\title{
Using a GPS-Aided Inertial System for Coarse-Pointing of Free-Space Optical Communication Terminals
}

\author{
Bernhard Epple* \\ German Aerospace Center (DLR), Institute of Communications and Navigation
}

\begin{abstract}
In the Capanina project, it has been shown that it is possible to use solely GPS positions for coarse-pointing of a stratospheric free-space optical communication terminal. Although this system design has been shown to be functional, it has to deal with acquisition and reacquisition times of up to 20 seconds. Whenever the line of sight is blocked, the Capanina terminal has to do a reacquisition of the partner terminal.

In land-mobile systems, e.g. transmission between a moving vehicle and a fixed station, where objects like trees or buildings can frequently block the line of sight, this type of acquisition/reacquisition is not acceptable.

In this paper a revised system design will be shown, which reduces the initial acquisition time to below a second by using information from a GPS-Aided Inertial System (GPS-INS). It will also be shown how reacquisition times and coarse-pointing errors can be reduced to a minimum by using feedback from the optical fine-pointing device. Finally a demonstrator setup and some test results will be presented.
\end{abstract}

Keywords: GPS, INS, PAT, AHRS, pointing, acquisition and tracking

\section{INTRODUCTION}

One of the most important abilities of a mobile optical communication terminal is to accurately locate and acquire (point towards) its counter terminal. Short acquisition and reacquisition times are main design requirements for mobile optical communication terminals. For links in a land-mobile environment it is most likely that the optical beams are obscured temporary by e.g. trees or buildings. The blocking of the line of sight (LOS) does not only break the communication signal, it also blocks the LOS to the beacon of the counter terminal. With a blocked LOS it is not possible to operate in a highly accurate optical fine-pointing mode. In this case an improvement of coarse pointing vectors (CPV) by measuring pointing errors with the fine-pointing assembly (FPA) is not possible. Hence, inaccuracy in the coarse pointing vectors will enhance reacquisition times.

For pointing a terminal towards a counter terminal, the terminal has to determine its own location and orientation and it has to know the location of the counter terminal. The location of the counter terminal can be predetermined or transmitted by the counter terminal, using a radio frequency low-data-rate signaling link, at run time. To determine location and orientation of a mobile terminal, special sensors have to be used, since these values are continuously changing and can not be predetermined prior run time, as it is possible for example for satellite tracking.

During the Capanina STROPEX trial it has been shown that it is possible to use only GPS positions of the counter terminal, transmitted via signaling link, for acquisition of a partner terminal [1]. The duration for an acquisition or a reacquisition run during this trial was up to 20 seconds and whenever the line of sight had been blocked, the Capanina terminal had to do a reacquisition of the counter terminal. With its long acquisition and reacquisition times the terminal from the Capanina project can not be used in a land-mobile environment, so a different type of pointing, acquisition and tracking (PAT) mechanism has to be developed for this field of operation.

\footnotetext{
*Bernhard.Epple@dlr.de; phone +49 (0) 8153 2816; +49 (0) 8153 2844; www.dlr.de/kn/

Copyright 2006 Society of Photo-Optical Instrumentation Engineers. This paper was published in Proceedings of the SPIE Vol. 6304 and is made available as an electronic reprint with permission of SPIE. One print or electronic copy may be made for personal use only. Systematic or multiple reproduction, distribution to multiple locations via electronic or other means, duplication of any material in this paper for a fee or for commercial purposes, or modification of the content of the paper are prohibited.
} 
The demonstrator terminal, named Mobile Optical Communication Terminal (MOCT), described in this paper uses a commercial GPS-INS as data source for its coarse-pointing assembly (CPA), since such systems are commonly used in avionic applications where it is also important to determine its own location and orientation. As data source, an attitude and heading reference system (AHRS) could also have been used, but the GPS-INS also measures velocities which could be of advantage for future use. Because off-the-shelf GPS-INSs are only accurate enough to be used for coarse-pointing an optical terminal, an additional fine-pointing assembly has to be added to the terminal for achieving the accuracy needed in optical communications (which is in the range of some mrad accuracy). Any type of active pixel array (APA), like a four-quadrant detector or a camera, can be used as data source for the calculation of the fine-pointing vector (FPA).

\section{MOCT System Overview}

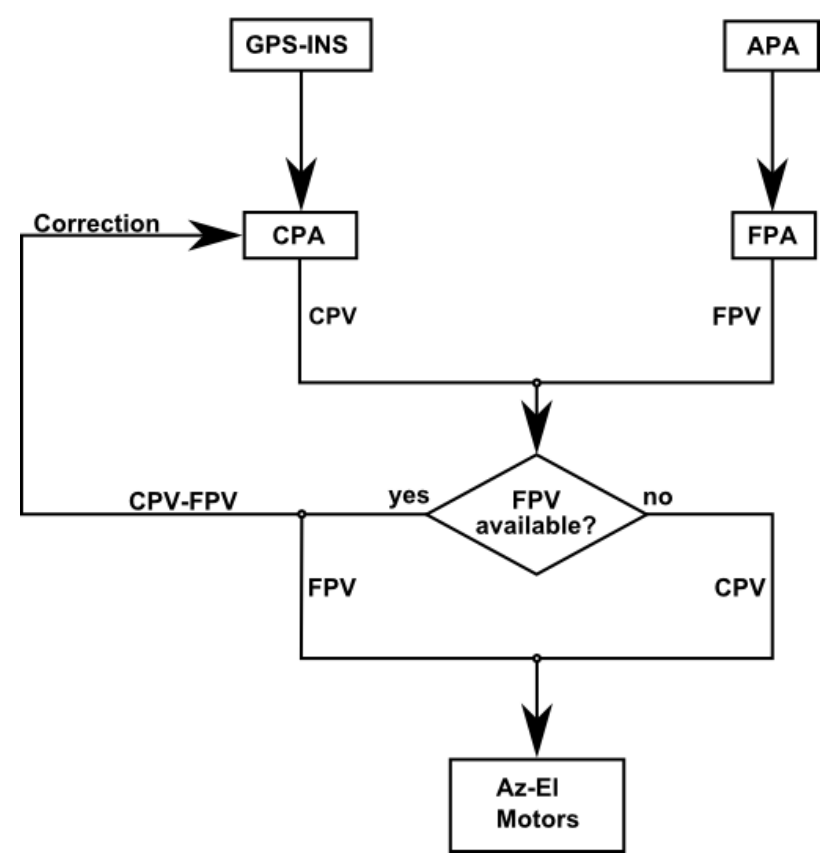

Fig. 1. System overview of the MOCT showing the interaction between FPA and CPA.

\section{THE DEMONSTRATOR}

The scenario used for the demonstrator described in this paper contains the MOCT mounted on a van which drives along a road about $1500 \mathrm{~m}$ away from the optical ground station (OGS) [2] based on the roof top of a DLR building. For the final tests, not described in this paper, the MOCT will transmit live images as test data to the OGS using intensity modulated $1550 \mathrm{~nm}$ communication system. The maximum velocity of the van carrying the terminal is set to be $60 \mathrm{~km} / \mathrm{h}$.

The task of the coarse-pointing assembly is the calculation of the pointing vector from MOCT to OGS by using the gathered positioning data and the data from the GPS-INS. By using an accurate GPS-INS the acquisition process of the partner terminal can be reduced to the calculation of the pointing vector between the terminals without any type of scan. This approach reduces the acquisition time to a minimum and therefore it is superior to systems which only use positioning information and some scanning to find its partners, as it is the case for the Capanina FELT terminal. An additional requirement for a scanless acquisition is, that the divergence angles of the used beacons and the field of views of the used detectors/cameras have to match the inaccuracy contained in the calculated pointing vector. Most important, the coarse-pointing assembly has to be accurate enough for moving the beacon of the counter terminal into the field of view of the fine-pointing assembly. 


\subsection{Pointing Vector Errors and their Impact on System Accuracy}

Since the coarse-pointing assembly is using data from two subsystems (GPS and AHRS), the measurement errors made by these systems will result in a faulty calculation of the pointing vector. The first error is caused by wrong position measurements. This also applies for systems without a GPS-INS. The second error is the error contained in the orientation measurements made by the GPS-INS.

\subsubsection{Position Measurement Error}

For the demonstrator a scenario has been chosen where the position of the partner terminal, the OGS, is well known and has been verified during several testing campaigns. Since the MOCT is a mobile system, its position has to be measured during run time. The error contained in this position measurement will be contained in the calculation of the pointing vector. The quality of the positioning data depends on the chosen hardware as shown in the following table, which gives an overview of expectable accuracies for different types of GPS receivers. These values can be assumed as "worst-case" accuracies because modern receivers use various optimization techniques to obtain better results. Since the demonstrator is using off-the-shelf GPS for position determination, all values are given referring to this system.

Table 1. 95 \% Expectable GPS accuracy (radius) for various receiver types [3].

\begin{tabular}{|l|l|}
\hline GPS accuracy with activated Selective Availability (SA) & $100 \mathrm{~m}$ \\
\hline Typical accuracy without SA & $15 \mathrm{~m}$ \\
\hline Typical accuracy for Differential GPS & $3-5 \mathrm{~m}$ \\
\hline Typical accuracy with WAAS/EGNOS & $1-3 \mathrm{~m}$ \\
\hline
\end{tabular}

The manual of the chosen GPS-INS states a position accuracy of $3 \mathrm{~m}$ circular error probable (CEP), and measurements have shown that the CEP is about $2.5 \mathrm{~m}$ and the $95 \%$ GPS accuracy radius is about $5 \mathrm{~m}$.

The impact of this error depends on the distance between the two terminals. The further the two terminals are away from each other the less the impact is. For example, if the distance between the two terminals is $500 \mathrm{~m}$ and the positioning error is $5 \mathrm{~m}$, the resulting error in the pointing vector will be $10 \mathrm{mrad}$. If the distance between the two terminals is increased to $5000 \mathrm{~m}$ the resulting error in the pointing vector will only be $1 \mathrm{mrad}$, and so on. Because of the decreasing pointing error for longer distances, the GPS error is neglectible in aeronautical scenarios, where at least 5400 kilometres of distance will be required by air traffic regulations between the two terminals.

Another error source in the position measurement is the update rate of the position receiver. The error can be calculated by multiplying the time since last measurement with the mean velocity since last measurement. Some example values are given in Table 2. The values for the velocity of $900 \mathrm{~km} / \mathrm{h}$ are given, because this is the general assumed cruising speed over ground of an Airbus A380. The maximum position error of 250 meters for this speed and an update rate of $1 \mathrm{~Hz}$ is very large. When converted to the angular error at the minimal distance of 5400 it becomes a maximum pointing error of about $2.6^{\circ}$. So for optical communications in an aeronautical environment, position receivers with a higher update rate should be used. The selected GPS-INS for the demonstrator has an update rate of $100 \mathrm{~Hz}$, so this error source can be ignored for the system design. The following table gives a few example error values for different update rates and velocities.

Table 2. Position error related to update rate o the receiver and the velocity of the receiver.

\begin{tabular}{|l|l|l|}
\hline Mean Velocity $(\mathrm{km} / \mathrm{h})$ & Position Receiver Update Rate $(\mathrm{Hz})$ & Maximum Position Error $(\mathrm{m})$ \\
\hline 10 & 1 & 2.7 \\
\hline 10 & 5 & 0.6 \\
\hline 10 & 100 & 0.03 \\
\hline 50 & 1 & 13.9 \\
\hline 50 & 5 & 2.8 \\
\hline
\end{tabular}




\begin{tabular}{|l|l|l|}
\hline 50 & 100 & 0.14 \\
\hline 900 & 1 & 250 \\
\hline 900 & 5 & 50 \\
\hline 900 & 100 & 2.5 \\
\hline
\end{tabular}

\subsubsection{Orientation Measurement Error}

Commercial GPS-INS are generally sensitive to the environment in which they are used, because in most cases they use a magnetometer as heading sensor which can easily be irritated by large ferrous or magnetic material objects. The measurement of the magnetometer also relies on a magnetic model of the earth which is not exactly representing the current state of the earth's magnetic field. A land-mobile environment, where several different objects can influence the magnetometer measurements, can be seen as worst case for such systems. For example in. aeronautical or nautical environments should be fewer disturbances and therefore, the measured heading angle should be more precise.

Although the magnetometer can be calibrated in its operational environment, the sensor will always produce small measurement errors, since the magnetic environment is not completely stable over time. The chosen GPS-INS has an rms heading error of $3^{\circ}$ measured in an aeronautical environment, which has a huge impact on the final system design. Compared to this value, the given error of $<0.75^{\circ} \mathrm{rms}$ for the roll and pitch sensor seem neglectible. The next section explains how the pointing error influences the system design of an optical terminal.

\subsection{Pointing Vector Error of the MOCT}

To determine the error contained in the pointing vector of the MOCT, some measurements have been done on the DLR premises in Oberpfaffenhofen. For these measurements the MOCT was mounted on a van which drove some circles and straight lines (see Ground Track below) in a distance of about 320 to 440 meters away from the OGS. The reference pointing vector (RPV) has been determined by pointing a beacon from the OGS towards the MOCT and using a camera on the MOCT as fine-pointing assembly. The image from the camera could be used for optical tracking of the beacon and therefore for obtaining the RPV. This camera-measured reference pointing vector was not the ideal pointing vector, because it contained the error of FPA, but it was good enough as reference for comparison with the CPV calculated from the GPS-INS data.

The measurements have shown that the mean pointing error for the azimuth axis is $2.39^{\circ}$ with a standard deviation (STD) of $1.12^{\circ}$ and for the elevation axis $0.3^{\circ}$ with a STD of $0.2^{\circ}$. During the tests it has been observed that the error on the azimuth axis seems to have a constant offset of $2^{\circ}$. The source for this offset is most likely an improper calibration of the GPS-INS. If a constant offset value of $2^{\circ}$ for the azimuth axis is introduced into the system, the mean pointing error on the azimuth axis goes down to $0.79^{\circ}$ and the STD goes slightly up to $1.25^{\circ}$. The gathered information will later be used for the optical system design. 


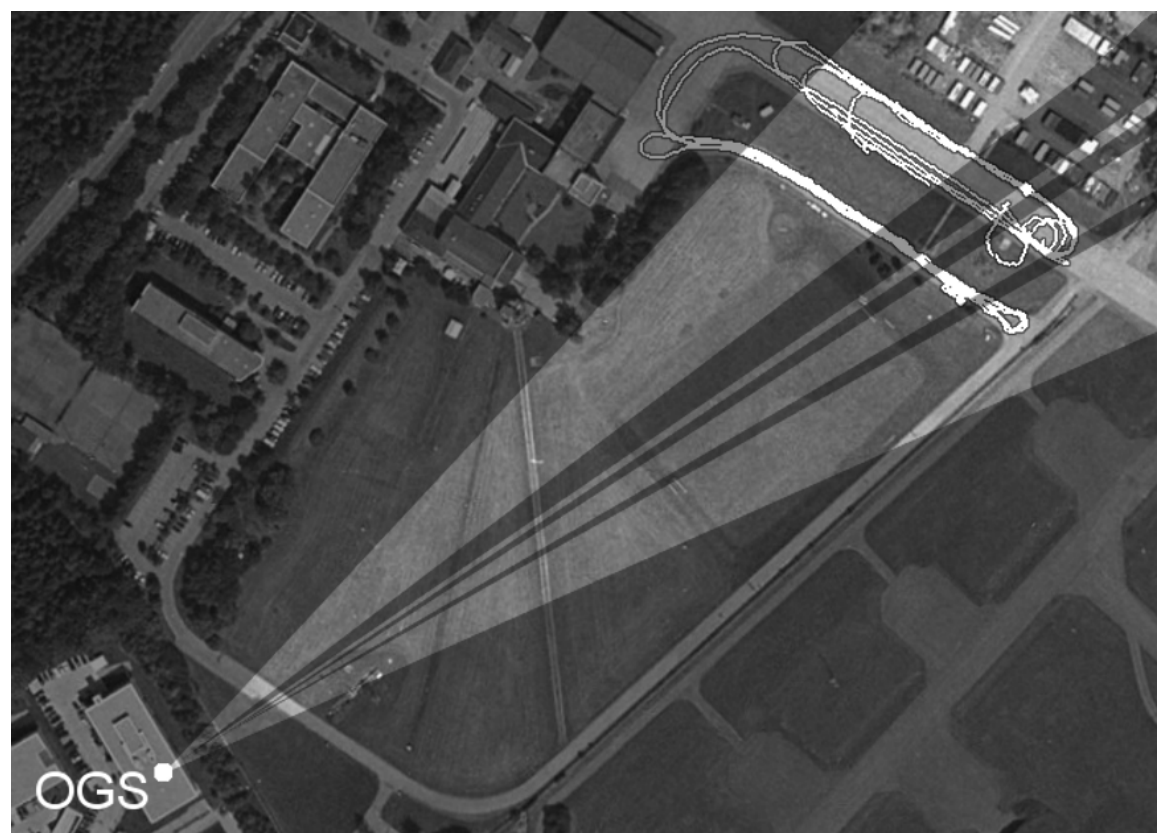

Fig. 2. Ground track of MOCT (white path upper right corner) during measurements done on the DLR premises. Parts of the track shadowed by obstacles in the line of sight from the OGS (lower left corner) are darkened in the image.

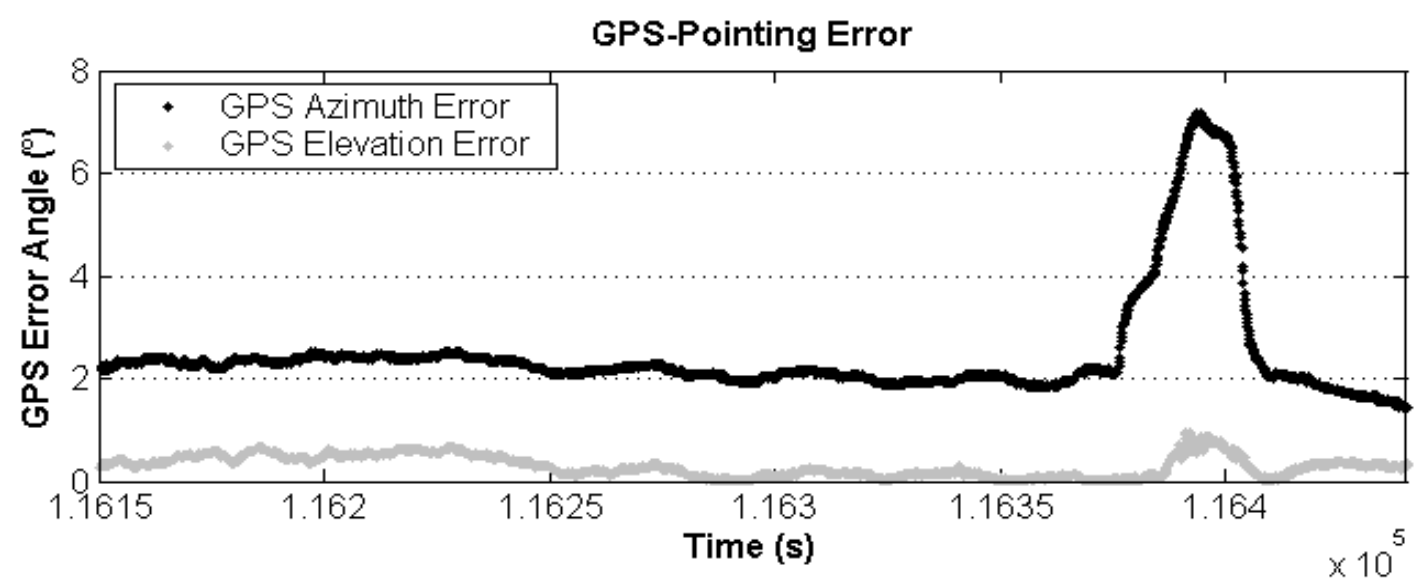

Fig. 3. Measurement example of the MOCT driving a $360^{\circ}$ loop. While this data was recorded, the van was driving approximately $500 \mathrm{~m}$ straight with a velocity of about $10 \mathrm{~km} / \mathrm{h}$. Then it was slowing down and driving a $360^{\circ}$ loop. The peaks in the error graphs during the turn result from inaccuracies of the GPS-INS during the turn. The error peak on the azimuth axis is caused by the turn itself, while the error peak on the elevation axis is caused by the van leaning outside the turn.

\subsection{Demonstrator System Design}

The error contained in the CPV is an important measure for deciding about the divergence angles of the used lasers and the field of views of the used detectors, cameras, etc. For a successful acquisition without the need for a scan, the divergence angles of the terminal beacons should be made large enough to illuminate the whole area of the pointing inaccuracy defined by the previous mentioned errors.

The pointing vector error causes the terminal not to point exactly on the target, but on a circle around the target. The radius of the circle at the target depends on the pointing vector error; for GPS-pointing between an object with known 
position and one with a GPS-measured position, the $95 \%$ error radius of the used GPS receiver can be used as radius of the circle. This circle will be called error circle throughout this paper.

The following figures will illustrate how a suitable divergence angle for the beacon can be determined. Fig. 4 shows that although the FWHM beam diameter is smaller than the area of position inaccuracy, the beacon can illuminate the whole error circle because the real beam is much broader than the FWHM diameter (assuming a Gaussian beam). Nevertheless the received power goes down by more than $50 \%$ if the target is outside the FWHM diameter.

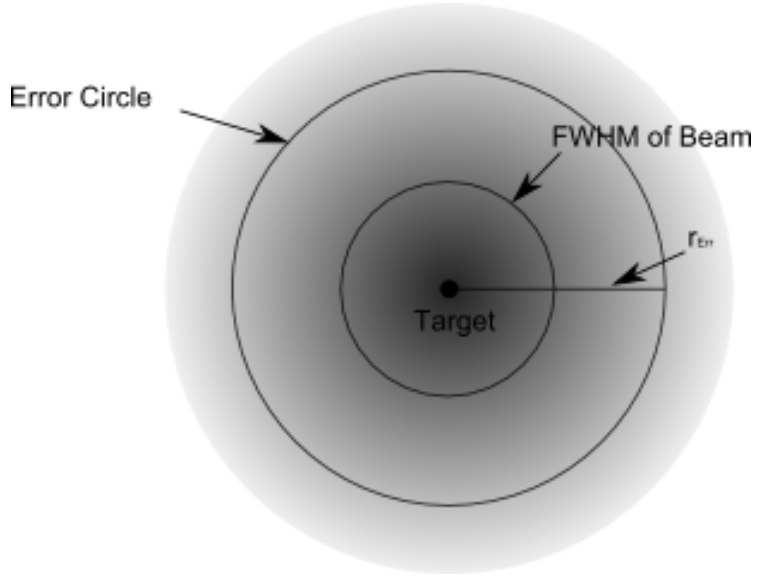

Fig. 4. Schematic overview of the illumination of the pointing target when no pointing error is present and the divergence angle of the beam is well chosen.

When a maximum error is contained in the position information, the target will receive some illumination. Since here at DLR all calculations for the link budget are typically done based on the FWHM divergence angle of the beacon, the received signal might not be strong enough for communication, but it might be strong enough for the fine-pointing assembly of the counter terminal to detect the beacon and adjust its pointing. So the divergence angle of the beacon has to match the pointing error of the coarse-pointing assembly and the sensitivity of the fine-pointing assembly of the counter terminal.

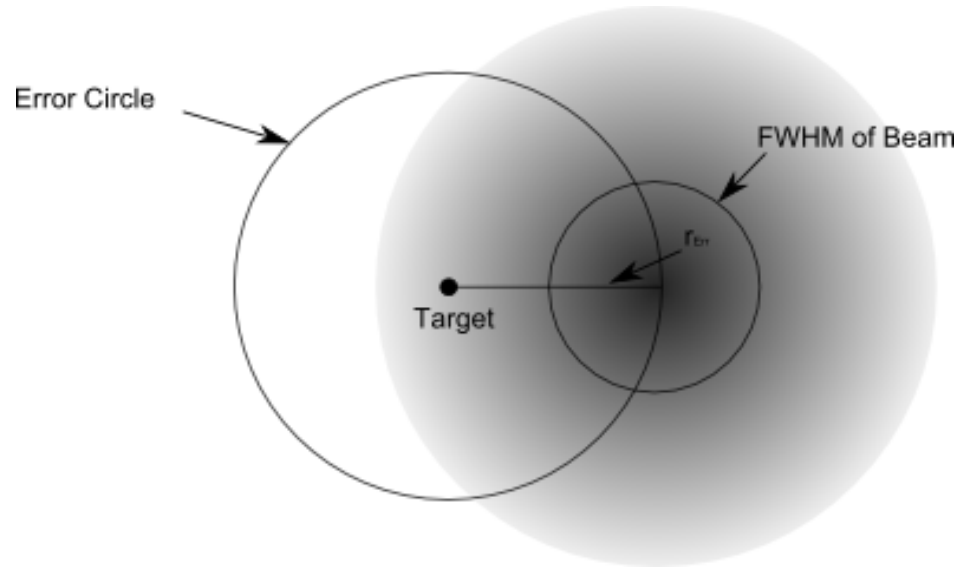

Fig. 5. Schematic overview of the illumination of the pointing target when the pointing error is at its maximum and the divergence angle of the beam is well chosen.

Another prerequisite for a successful acquisition is that the field of view of the fine-pointing assembly of the terminal is at least as large as the maximum error contained in its CPV. Otherwise, in worst case, the FPA will not be able to detect the beacon of the counter terminal even if the beacon is perfectly targeted on the terminal. How the mentioned factors influence the design of the optical system, will be shown in the following with the demonstrator setup as an example.

In the demonstrator scenario there is the OGS, of which the global position and orientation is well known. Therefore the pointing error of the OGS depends only on the error contained in the GPS positions. With this in mind the smallest 
suitable FWHM divergence angle $\left(\mathrm{FWHMdiv}_{\min }\right)$ for the beacon of the partner terminal can be calculated using the following simplified formula, where $\mathrm{d}_{\min }$ is the minimum distance between the terminal and err $_{\mathrm{r}}$ is the error radius.

$$
\text { FWHMdiv }_{\min }=\frac{2 * e r r_{r}}{d_{\min }}
$$

In the demonstrator setup, the maximum GPS error was measured to be a radius of 5 meters and the minimum link distance between the two terminals has been chosen to be 1300 meters. With the formula above, this results in a minimum divergence angle of $11.54 \mathrm{mrad}$ for the OGS beacon. To be safe, a FWHM divergence angle of $15 \mathrm{mrad}$ has been chosen. The full field of view of the FPA of the OGS has been set to $\sim 0.6^{\circ}$.

For the MOCT, things are different. For this terminal, the error caused by the GPS is nearly neglectible compared to the error introduced by the heading sensor of the GPS-INS. With the given error of the heading sensor of $3^{\circ}$, the field of view of the fine-pointing assembly at the MOCT should be at least $6^{\circ}$. Using the results of the test measurements, the field of view should even be set to $14^{\circ}$ to be able to do an acquisition during turns. For the demonstrator it has been decided not to start the acquisition during a turn. Because the measurements have shown that it is reasonable to expect a pointing error on the azimuth axis of less than $1^{\circ}$, when using an offset on the azimuth axis, a field of view of $2^{\circ}$ should be sufficient for the FPA. For the demonstrator FPA a field of view of $2.7^{\circ}$ has been implemented, as this could be built with available components. This decision means that the demonstrator will not be perfect for land-mobile use, but suitable for the given demonstration scenario. If the acquisition should be started during a turn it might be successful. If not, it will be successful after the turn. The transmission laser of the MOCT is set to have a divergence angle of $15 \mathrm{mrad}$ and it will also be used as beacon for the FPA of the OGS.

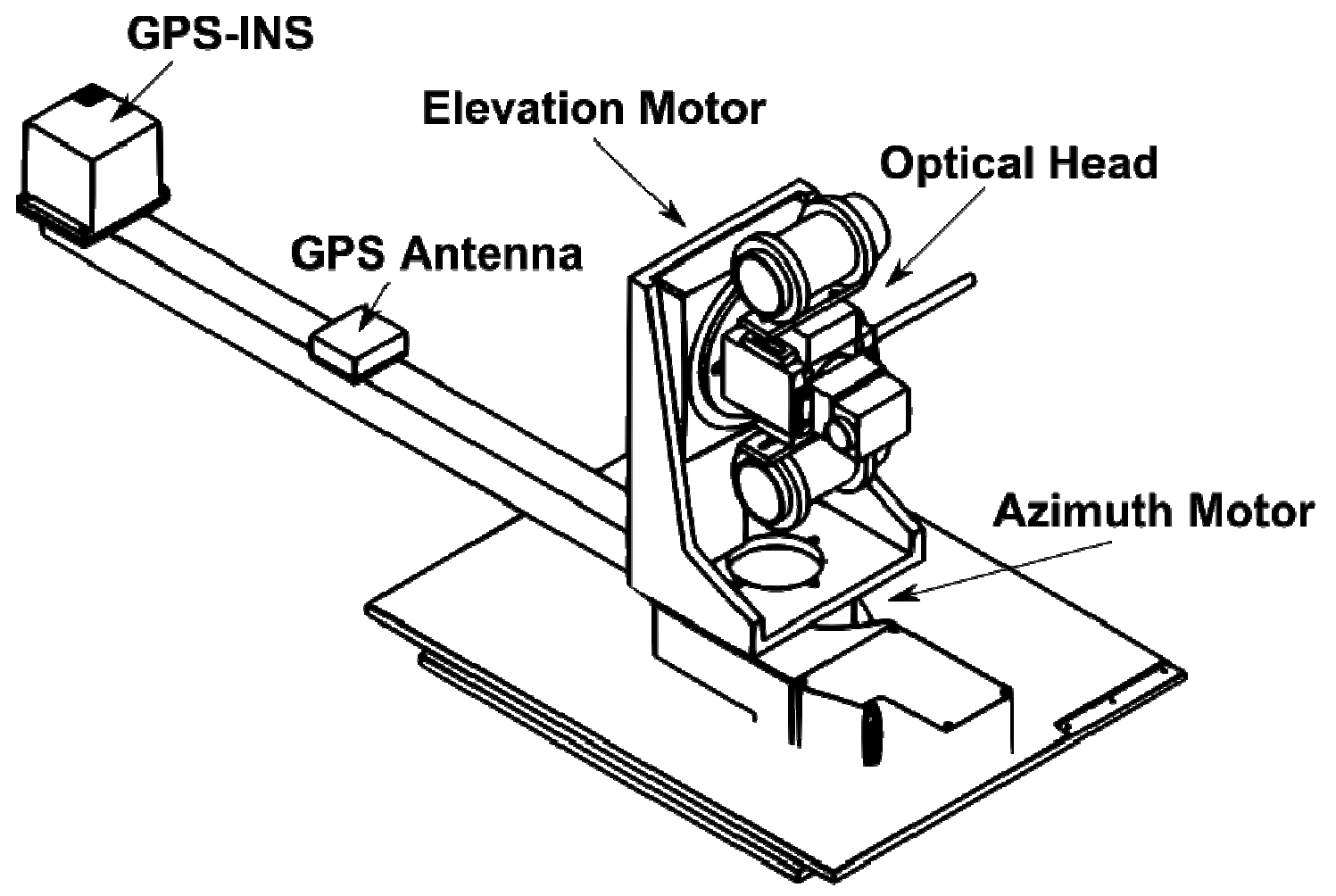

Fig. 6. Schematic overview of the mobile optical communication terminal (MOCT). The GPS-INS is mounted on a boom to get enough distance between the unit and the magnetic fields of the motors.

\section{OPTIMIZATION OF THE REACQUISITION PROCESS}

A fast reacquisition of a broken link is an important precondition for robust optical communications. In scenarios where broken links are very rare, this might seem not so important, because e.g. after a broken link for 20 seconds, there might 
be data transmission for a few hours. But in scenarios where the link might break every minute or so, it is important to keep the duration of the link breakage as short as possible.

The need for reacquisition or is given when, after a blocked line of sight, the beacon of the counter terminal is no longer visible on the detector of the fine-pointing assembly. In the demonstrator scenario this means that the pointing of the MOCT or the OGS becomes worse than $7.5 \mathrm{mrad}\left(0.43^{\circ}\right)$ and therefore the beacon of one of the two terminals is no longer visible for its counter terminal. For the OGS, the beacon divergence angle is designed to be slightly wider than the expected GPS-pointing error, so there should occur no problems at the OCG which would require a reacquisition. For the MOCT, the $0.43^{\circ}$ pointing accuracy are a tough requirement as we have learned above; the mean pointing error on the azimuth axis is $2.39^{\circ}$ and during turns it can even be more. The measured accuracy of $0.79{ }^{\circ}$ for the azimuth axis using a constant offset should be good enough for keeping the target beacon in the field of view of the FPA, but the coarse pointing will not be accurate enough to continue communication immediately after an obstacle has been passed. One method to achieve better coarse-pointing results for reacquisition is to use feedback from the fine-pointing assembly as a correction value. These corrections will only be valid for a short duration of a few seconds, but this does not matter because only short blockings with durations of less than one second are expected in our scenario. In the previously described measurement setup a permanently unblocked line of sight between the two terminals is needed for calculating the reference pointing vector. So it is not possible to block the LOS and directly measure the result of the optimization. Instead, the measurement data from the previous measurements could be taken as input for simulations. For this paper, the results of two optimization techniques have been simulated.

\subsection{Calculation of Axes Offsets}

For this type of optimization, the calculated pointing vector from FPA is taken as RPV and compared with the pointing vector calculated by the coarse-pointing assembly. The offset between the two vectors is stored in a variable which gets updated whenever the fine-pointing assembly detects a signal and calculates a new FPV. If the signal got lost, the coarsepointing assembly used the last calculated offset as correction offset for the CPV. Only one pointing vector from the fine-pointing assembly is needed to calculate the offset, so for simulations it has been assumed that the line of sight between the two terminals is mostly blocked and the offset can only be calculated in a fixed correction interval. The simulation results show a huge improvement of the overall GPS-pointing accuracy, especially for the expected blocking duration of one second. In Fig. 7 you can see that most of the time the coarse-pointing of the MOCT is accurate enough to immediately continue communication between the terminals. Only during the turn it might happen that the MOCT has to reacquire the OGS, but it can bee guaranteed, that the OGS will be visible to the fine pointing unit of the MOCT.

Table 3. Mean axis errors and their STD for different correction intervals

\begin{tabular}{|l|l|l|l|l|}
\hline $\begin{array}{l}\text { Correction } \\
\text { Interval (s) }\end{array}$ & $\begin{array}{l}\text { Mean GPS-Pointing } \\
\text { Error Azimuth }\left(^{\circ}\right)\end{array}$ & $\begin{array}{l}\text { STD GPS- } \\
\text { Pointing Error } \\
\text { Azimuth }\left(^{\circ}\right)\end{array}$ & $\begin{array}{l}\text { Mean GPS-Pointing } \\
\text { Error Elevation }\left(^{\circ}\right)\end{array}$ & $\begin{array}{l}\text { STD GPS- } \\
\text { Pointing Error } \\
\text { Elevation }\left(^{\circ}\right)\end{array}$ \\
\hline 1 & 0.065 & 0.37 & 0.03 & 0.06 \\
\hline 2 & 0.10 & 0.42 & 0.04 & 0.07 \\
\hline 5 & 0.23 & 0.65 & 0.07 & 0.10 \\
\hline 10 & 0.50 & 1.16 & 0.10 & 0.14 \\
\hline
\end{tabular}




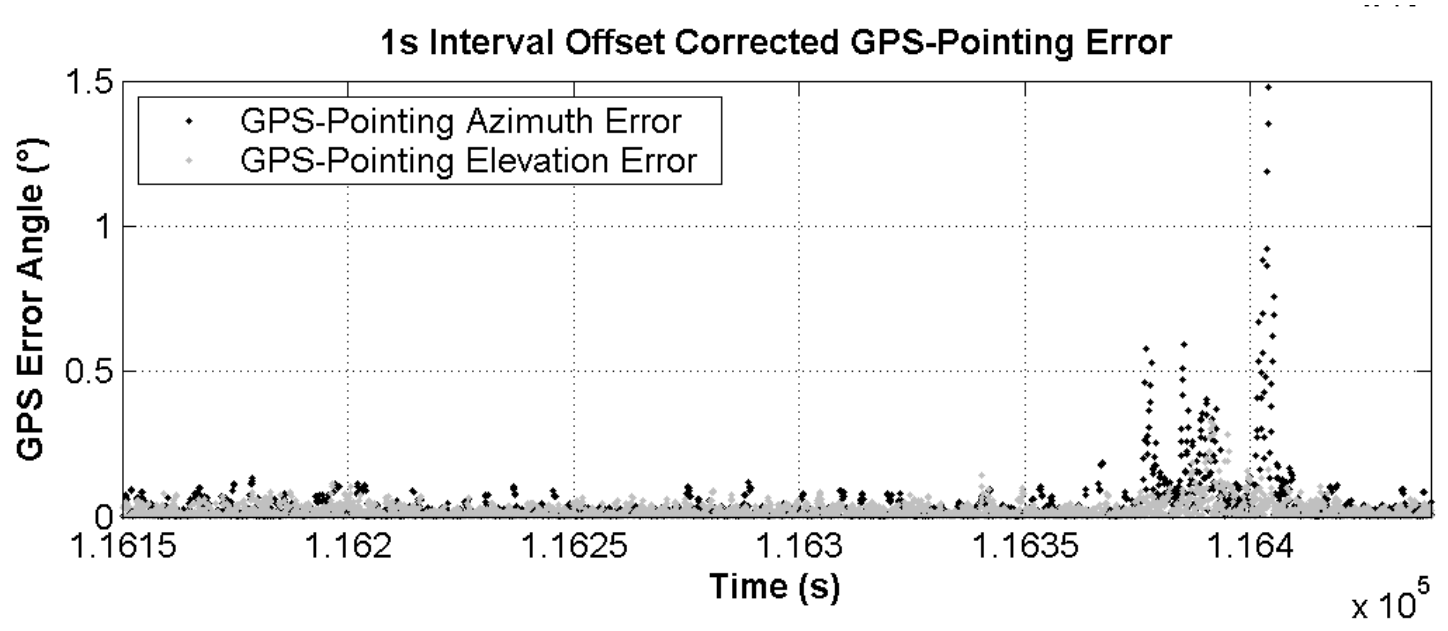

Fig. 7. This plot shows the calculated GPS-pointing errors for a correction interval of 1 second. Even during the turn, the errors stay below 1.5 degree which is a great improvement compared to the errors shown in Fig. 3.

\subsection{Filtering the Axes Positions}

A common approach for optimizing the results of tracking systems is the use of filtering techniques like g-h filters (also known as $\alpha-\beta$ filters) or Kalman filters [4]. For this paper it has been decided to do further research in the benefits of using a g-h filter on top of the results of the optimization described in the previous section. The use of a g-h filter is a two step approach for filtering the measurement results. The first step is taking a measurement of the current axes positions and the second step is predicting the next axes positions based on the previously made predictions and the current measurements. An important characteristic of these filters is that they operate iterative, i.e. they only have to store the last prediction made and the current measurement results, so they can be implemented with a constant memory complexity, which is important for systems that should operate over a long period of time. Another good characteristic of the used g-h Filter is that it is not only predicting the positions of the azimuth and elevation axis, but it is also predicting the velocities of the two axes. This prediction allows the system to be used with different types of axis motors.

In the demonstrator system, following formulae are used for predicting the next axes positions / velocities. The given formulae are actually the formulae used for the azimuth axis, but the same formulae are used for the elevation axis using equivalent variables.

$$
\begin{gathered}
p v A z=l p v A z+\frac{A z H}{d t} *(m A z-l p A z) \\
p A z=l p A z+A z G *(m A z-l p A z)+d t * p v A z
\end{gathered}
$$

Where:

$p v A z$ is the prediction of the azimuth axis velocity.

lpvAz is the last predicted velocity of the azimuth axis.

$A z G$ is a constant factor.

$\mathrm{AzH}$ is a constant factor.

$d t$ is the time since the last prediction has been made.

$m A z$ is the current measured position of the azimuth axis.

$p A z$ is the prediction of the azimuth axis position.

$\operatorname{lp} A z$ is the last predicted position of the azimuth axis. 
For the g-h filter, the factors $A z G$ and $A z H$ (respectively $E I G$ and $E l H$ for the elevation axis) have to be set before the operation of the filter. More sophisticated filters like the Kalman filter are able to calculate the optimal factors during run time, so such filters can be expected to give better results than the used g-h filter. The simulations have shown that the factors $A z G=0.84, A z H=0.36, E l G=0.99, E l H=0.96$ give good results for the simulated scenario. As measurement of the axes positions the FPV is used whenever possible, otherwise the CPV. So for long blockings, the filtered axes positions will degenerate to the accuracy of the original CPV calculated solely from GPS-INS data.

Since the filter needs several measurement values to operate satisfactorily, and not just one single measurement like the previous optimization does, the simulated scenario has been adjusted in the following way.

The line of sight between the two terminals is blocked every 30 seconds for duration of between 1 and 10 seconds. Since there might be some coherence in the simulation results between the trajectory and the occurrence of a blocked line of sight, the blockings have been simulated on various parts of the measurement track, but this did not result in significant changes in the simulation results. The simulation results are given in the table and graph below, where you can see that the CPA is able to keep the tracking target constantly in the field of view of the FPA. For an assumed maximum blocking duration of one second the system is now accurate enough to keep the communication beam in most cases targeted on the receiver terminal. The last few cases where communication can not continue immediately after the obstacle has been passed should be eliminable by the use of a Kalman filter.

Table 4. Mean axis errors and their STD for different blocking durations

\begin{tabular}{|l|l|l|l|l|}
\hline $\begin{array}{l}\text { Blocking } \\
\text { Duration (s) }\end{array}$ & $\begin{array}{l}\text { Mean CPA-Pointing } \\
\text { Error Azimuth }\left(^{\circ}\right)\end{array}$ & $\begin{array}{l}\text { STD CPA- } \\
\text { Pointing Error } \\
\text { Azimuth }\left(^{\circ}\right)\end{array}$ & $\begin{array}{l}\text { Mean CPA-Pointing } \\
\text { Error Elevation }\left(^{\circ}\right)\end{array}$ & $\begin{array}{l}\text { STD CPA- } \\
\text { Pointing Error } \\
\text { Elevation }\left(^{\circ}\right)\end{array}$ \\
\hline 1 & 0.03 & 0.11 & 0.02 & 0.03 \\
\hline 2 & 0.04 & 0.12 & 0.02 & 0.04 \\
\hline 5 & 0.11 & 1.37 & 0.03 & 0.07 \\
\hline 10 & 0.24 & 1.54 & 0.05 & 0.10 \\
\hline
\end{tabular}

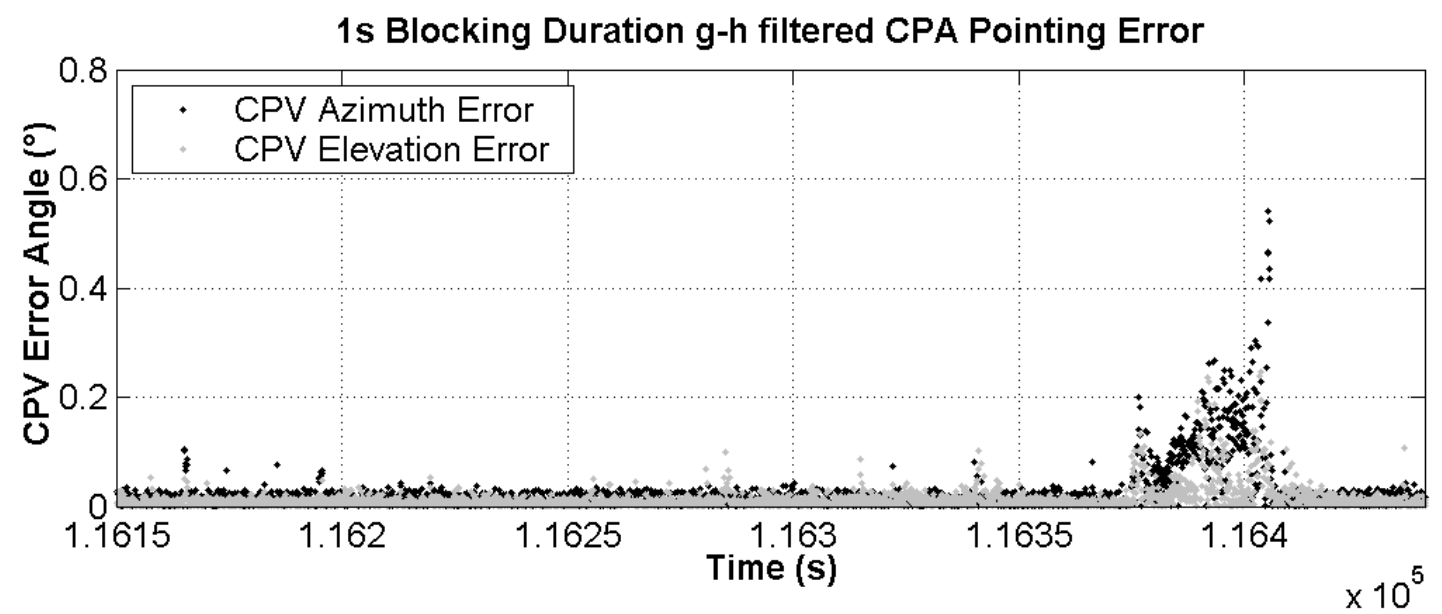

Fig. 8. This plot shows the calculated CPV errors for a blocking duration of one second. Even during the turn, the errors stay mostly below 0.43 degree which means that communication can continue immediately after the obstacle has been passed and no reacquisition is needed. 


\section{CONCLUSION}

The demonstrator described in this paper has shown that it is possible to build a land-mobile optical communication terminal using solely off-the-shelf components. The terminal is designed in a way that no special steps are required prior start of the acquisition phase. After the system has started up, the terminal is immediately ready to use. This is a big advantage over systems which have to complete a long calibration process before they are ready to use, e.g. the OGS. The design of the MOCT also allows using the demonstrator terminal not only for the purpose of optical communications; for example the demonstrator has also successfully been used for star and satellite tracking.

A future point of research will be to enable the terminal to complete the acquisition phase during a turn of the carrying vehicle. This can be done by using a more accurate AHRS, or by implementing a more complex initialization process, or by implementing a larger field of view for the FPA of the terminal.

\section{ACKNOWLEDGEMENT}

I want to thank Hennes Henniger and Dr. Nicolas Perlot (both DLR) for their valuable contribution and assistance especially preparing simulations and measurements.

\section{REFERENCES}

[1] J. Horwath, M. Knapek, B. Epple, M. Brechtelsbauer, B. Wilkerson, "Broadband Backhaul Communication for Stratospheric Platforms: The Stratospheric Optical Payload Experiment (STROPEX)", to be published in Proceedings of the SPIE 2006, Vol. 6304.

[2] M. Knapek, J. Horwath, N. Perlot, K. Zettl, D. Giggenbach, “Transportable Optical Ground Station for Free-Space Laser Communications”, Proceedings of the $3^{\text {rd }}$ Advanced Satellite Mobile Systems Conference (ASMS 2006), Herrsching, May 2006.

[3] Dr. A. Köhne, Dr. M. Wößner, http://www.kowoma.de/gps/Genauigkeit.htm.

[4] E. Brookner, Tracking and Kalman Filtering made easy, John Wiley \& Sons, New York, 1998. 